sciendo

DOI: $10.2478 /$ BIPMF-2021-0003
BULETINUL INSTITUTULUI POLITEHNIC DIN IAŞI

Publicat de

Universitatea Tehnică „Gheorghe Asachi” din Iaşi Volumul 67 (71), Numărul 1, 2021

Secţia

\title{
PERTURBED KEPLERIAN BEHAVIOR IN SPIRAL GALAXIES DESCRIBED BY THE MANNHEIM-KAZANAS METRIC
}

\author{
VITALIE LUNGU ${ }^{1, *}$ and ANDROMEDA-IUSTINA DARIESCU ${ }^{2}$ \\ 1"Alexandru Ioan Cuza" University of Iaşi, \\ Faculty of Physics, Iaşi, Romania \\ 2“Alexandru Ioan Cuza" University of Iasi, \\ Faculty of Mathematics, Iaşi, Romania
}

Received: February 17, 2021

Accepted for publication: March 31, 2021

\begin{abstract}
The aim of this paper is to analyze the Gordon equation for the Mannheim-Kazanas metric which contains, besides the Schwarzschild solution, two other additional contributions. This metric is offering an explanation for the flat galactic velocity curves, without requiring dark matter. The test particle orbiting the galaxy center will feel not only the matter of the Galaxy, but also the cosmological background which is acting like a perturbation.
\end{abstract}

Keywords: Mannheim-Kazanas Spacetime; Spiral galaxy; Black Holes; Gordon equation; Heun functions.

\section{Introduction}

For spiral galaxies, the so-called rotation curves, defined as the circular velocities around the nucleus, are the basic tool for deriving the mass distribution. Observations performed more than forty years ago, have shown that the tangential velocity as a function of the equatorial radius does not follow the familiar Keplerian decline (Rubin et al.,1980). Thus, instead of decreasing

*Corresponding author; e-mail: vitalie.lungu97@mail.ru

This is an Open access article licensed under the Creative Commons Attribution-Non Commercial - No Dericatives 4.0 International License (CC BY-NV-ND 4.0) 
with the square root of the radius, the rotation curves are universally flat for massive galaxies and are monotonically increasing for the less massive ones (Persic and Salucci, 1995).

The difference between the galaxy mass given by the luminosity and the mass predicted by the rotation velocities has been seen as a strong evidence for the existence of spherical haloes of dark matter surrounding the spiral galaxies and substantial efforts have been directed towards detecting this type of matter.

In the same time, motivated by the lack of tangible evidence for the existence of the dark sector, different theories have been proposed, starting with the modification of the Newtonian dynamics introduced by Milgrom (1983).

As an alternative to Einstein's General Relativity, the Conformal Gravity (CG) is a reliable candidate and, similarly to Einstein's General Relativity, it uses a metric to describe the curved spacetime. One of the most promising results in CG is the Mannheim-Kazanas (MK) vacuum solution (Mannheim and Kazanas, 1989), because it provided a geometrical explanation for the flat galactic rotational curves (Mannheim and O'Brien, 2012). The MK metric contains a linear contribution, $\gamma r$, which has to be comparable with $2 \mathrm{M} / \mathrm{r}$ to effectively raise the rotation curves above Newtonian gravity predictions.

In the last years, the MK metric was tested on a large number of galaxies, including the dwarf ones, which are considered suitable targets for indirect dark matter detection (Ahnen et al., 2018). In this respect, the MK metric has provided fully acceptable fits, without the need of dark sector (O’Brien and Mannheim, 2012).

The last part of our work is dedicated to the Gordon equation. The massive bosons can be seen as test fields evolving in the MK spacetime. In the case of the Schwarzschild metric, the Gordon equation is satisfied by the Heun confluent functions (Vieira and Bezerra, 2016). The particle's quantized energy spectrum is related to the quasinormal modes and is completely determined by the black hole's mass. However, in the case of the MK metric, the Gordon equation does not admit an analytical solution and one has to use a perturbative approach.

\section{The Mannheim-Kazanas Solution}

The Mannheim-Kazanas (MK) metric is a vacuum solution of the equations of conformal gravitation and has the expression (Mannheim and Kazanas, 1989):

$$
g_{00}=1-\frac{2 \beta}{r}+\gamma r-\lambda r^{2} \text {, }
$$


where $\beta, \gamma$ and $\lambda$ are integration constants. With $\beta=M$ in the expression (1), one may notice that linear and quadratic contributions have been added to the familiar Schwarzschild metric.

In the original work of Mannheim and Kazanas (Mannheim and Kazanas, 1989), the value of the positive constant $\gamma$ was considered comparable to the inverse of the Hubble length. Later, it has been taken as $\gamma=\gamma_{0}+\gamma_{G}$, where $\gamma_{0}=3 \times 10^{-28} \mathrm{~m}^{-1}$ and $\gamma_{G}=N^{*} \gamma^{*}$, with $N^{*}=M / M_{S}$, $\gamma^{*}=5.42 \times 10^{-39} \mathrm{~m}^{-1}, M_{S}$ is the solar mass and $M$ is the galaxy mass. The parameter $\lambda$, whose value is $\lambda=9.54 \times 10^{-50} \mathrm{~m}^{-2}$, is universal for all galaxies and is due to the inhomogeneous material in the Universe (Mannheim and O'Brien, 2012).

By neglecting the $\lambda$ term, one may write the metric (1) in the form:

$$
g_{00}=1-\frac{2 M}{r}+\gamma r
$$

where the linear term proportional to the radius is the one capable to explain the flat galactic rotational curves, in the outer region, where the Newtonian term is falling off. On the other hand, for the Sun whose distance from the Galactic Center is $R_{0} \approx 8 \mathrm{kpc}$, the term proportional with $\gamma$ is very small and it cannot compete with the Newtonian contribution.

For $N^{*}$ solar masses in the luminous galaxy whose mass is given by $M=N^{*} M_{S}=(M / L) L$, the potential is computed by integration over the visible galactic mass distribution.

For the particular metric (2), one can write down the following expression of the potential:

$$
V=-\frac{M}{r}+\frac{\gamma r}{2}
$$

where

$$
\gamma=\gamma_{0}+N^{*} \gamma^{*}=\gamma_{0}\left[1+\frac{M}{M_{0}}\right]
$$

with

$$
M_{0}=\frac{\gamma_{0}}{\gamma^{*}} M_{S}=5.6 \times 10^{10} M_{S} .
$$

Thus, for galaxies with $M<<5.6 \times 10^{10} M_{S}$, we may consider $\gamma \approx \gamma_{0}$.

As it is known, the circular velocity at the galactic plane can be computed as: 


$$
v^{2}=r \frac{d V}{d r}
$$

where the gravitational potential, $V$, has multiple components, namely the central black hole, the bulge, the disk and the dark halo. However, one has to find a suitable mass distribution function to agree with the observations.

In Newtonian gravity, the tangential velocity of a test particle in circular motion around the central mass has the simple expression:

$$
v=\sqrt{\frac{M}{r}} .
$$

In General Relativity, the effective potential and the velocity of a particle moving around the galactic center are derived from the timelike geodesics. Thus, we write the spacetimeline element as

$$
d s^{2}=\frac{(d r)^{2}}{g_{00}}+r^{2}\left[(d \theta)^{2}+\sin ^{2} \theta(d \varphi)^{2}\right]-g_{00}(d t)^{2}=-d \tau^{2}
$$

where $\tau$ is the proper time and $g_{00}$ is given in (1). For a particle in the equatorial plane $(\dot{\theta}=\dot{\varphi}=0)$ with the conserved energy

$$
E=g_{00} \dot{t},
$$

where dot means the derivatives with respect to $\tau$, the relation (6) becomes

$$
\dot{r}^{2}=E^{2}-g_{00} .
$$

By comparing the above relation with the expression of the equation of motion for a testparticle with unit mass,

$$
\frac{\dot{r}^{2}}{2}=\frac{E^{2}-1}{2}-V_{e f f},
$$

one can identify the effective potential

$$
V_{\text {eff }}=\frac{1}{2}\left[-\frac{2 M}{r}+\gamma r-\lambda r^{2}\right] .
$$

Thus, the velocity defined in (5) reads 


$$
v^{2}=r \frac{d V}{d r}=\frac{M}{r}+\frac{\gamma r}{2}-\lambda r^{2}
$$

where, for galactic systems, the effect of the linear term may be of the same order of magnitude with the Newtonian one. For a typical galaxy with $M=10^{11} M_{S}$, these two terms are almost equal for $r_{*}=\sqrt{2 M / \gamma} \approx 20 \mathrm{kpc}$. Thus, for the outermost regions, the linear term will become dominant.

In the case of the Milky Way which is hosting, in the Galactic Center, a massive black hole, the total mass, including the dark halo up to $150 \mathrm{kpc}$, is $M=3 \times 10^{11} M_{S}$ (Sofue, 2015). At such large distances, the contribution $\lambda r^{2}$ should also be taken into account.

Since $v^{2}$ should be a positive quantity, the ratio $\gamma /(2 \lambda)$ can be seen as a natural limit on the size of galaxies, in the sense that beyond this distance there could no longer be any bound galactic orbits. For a small galaxy with $\gamma=\gamma_{0}$, this expression is around $\gamma_{0} /(2 \lambda) \approx 50 \mathrm{kpc}$. One has to point out that measurements of the dynamical mass of dark halos in most galaxies are made at radii of $r \approx 20-30 \mathrm{kpc}$.

\section{The Gordon Equation}

Let us consider a test particle evolving in the spacetime endowed with the metric function (1), with $\beta=M$. The inclusion of the terms proportional to $r$ and $r^{2}$ in the Schwarzschild solution will weakly break the asymptotic flatness of the Schwarzschild metric resulting in a constant inward acceleration. In view of the proposed numerical values of $\gamma$ and $\lambda$, the terms $\gamma r$ and $\lambda r^{2}$ will come out to be order unity at the edge of the observable universe. Hence, at length scales much smaller than that, they can be treated as perturbations.

The corresponding horizons are given by the equation $g_{00}=0$, namely these are the solutions of the cubic equation:

$$
-\lambda r^{3}+\gamma r^{2}+r-2 M=0 .
$$

For the universal values of the parameters $\gamma$ and $\lambda$ proposed by Mannheim and Kazanas, the discriminant:

$$
\begin{aligned}
& \Delta=18 a b c d-4 b^{3} d+b^{2} c^{2}-4 a c^{3}-27 a^{2} d^{2} \\
& =-108 M^{2} \lambda^{2}+4(1+9 \gamma M) \lambda+\gamma^{2}(1+8 \gamma M)
\end{aligned}
$$


is a positive quantity and thus one has three real solutions. One of the two positive roots is close to the Schwarzschild horizon,

$$
r_{h} \approx 2 M[1-2 \gamma M],
$$

while the other one is the de Sitter-like cosmological horizon

$$
r_{c} \approx 1 / \sqrt{\lambda} .
$$

By solving the Gordon equation, one can find important properties of the astrophysical object. For values of $r>R$, the corresponding Gordon equation is

$$
\frac{1}{r^{2}} \frac{\partial}{\partial r}\left[r^{2} g_{00} \frac{\partial \Phi}{\partial r}\right]+\frac{1}{r^{2}} \Delta_{\theta, \varphi} \Phi-\frac{1}{g_{00}} \frac{\partial^{2} \Phi}{\partial t^{2}}-\mu^{2} \Phi=0,
$$

where

$$
\Delta_{\theta, \varphi} \Phi=\frac{1}{\sin \theta} \frac{\partial}{\partial \theta}\left[\sin \theta \frac{\partial \Phi}{\partial \theta}\right]+\frac{1}{\sin ^{2} \theta} \frac{\partial^{2} \Phi}{\partial \varphi^{2}} .
$$

One may employ the variables separation

$$
\Phi=R(r) Y_{l}^{m}(\theta, \varphi) e^{-i \omega t},
$$

where the radial function $R$ is the solution of the equation

$$
\frac{d}{d r}\left[r^{2} g_{00} \frac{d R}{d r}\right]+\left[\left(\frac{\omega^{2}}{g_{00}}-\mu^{2}\right) r^{2}-l(l+1)\right] R=0,
$$

where $g_{00}$ has the expression (1). The above equation has an analytical solution only in the massless case and for $\lambda=0$ (Dariescu and Dariescu, 2021).

The absolute value of the numerical solution of the Eq. (10) is represented in the Fig. 1. One may note the dominant maximum just outside the horizon and the presence of a plateau for large values of the radial coordinate.

In the case of the Schwarzschild metric, which is the typical static solution for the exterior spacetime of a galaxy with the mass parameter equal to its total mass, the Gordon equation can be exactly solved, its solution being given by the Heun confluent functions (Ronveaux, 1995; Slavyanov and Lay, 2000). 


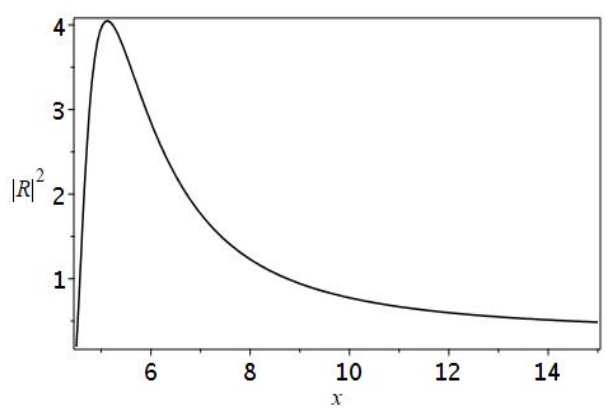

Fig. 1 - The absolute value of the solution of Eq. (10) for the MK metric.

For the metric function

$$
g_{00}^{S}=1-\frac{2 M}{r},
$$

the radial function, $R_{\mathrm{S}}$, has the expression

$$
R_{S}(r)=A e^{\frac{\alpha x}{2}} x^{\frac{\beta}{2}} \operatorname{HeunC}[\alpha, \beta, \gamma, \delta, \eta, x]
$$

where the Heun confluent functions have the variable $x=1-r /(2 M)$ and the parameters

$$
\begin{aligned}
& \alpha=-4 i \sqrt{\omega^{2}-\mu^{2}}, \beta= \pm 4 i M \omega, \gamma=0, \\
& \delta=4 M^{2}\left(\mu^{2}-2 \omega^{2}\right), \eta=-\delta-l(l+1) .
\end{aligned}
$$

By inspecting the Fig. 2, where the absolute value of the relation (11) is represented, one may notice a series of decreasing maxima, as the variable $x$ is increasing. Unlike the previous case, the radial function has an oscillatory behavior, with zero minima.

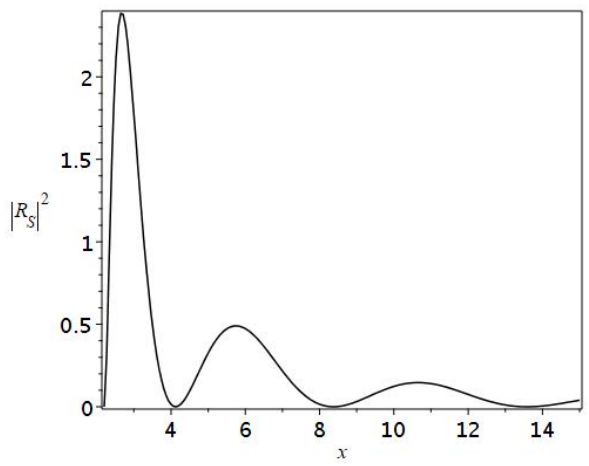

Fig. 2 - The absolute value of (11) for the Schwarzschild metric. 
For the asymptotic behavior at large values of $r$, where the linear and quadratic terms must be taken into account as perturbations, one may use the formula (Slavyanov and Lay, 2000)

$$
\begin{aligned}
& \text { HeunC }[\alpha, \beta, \gamma, \delta, \eta, x] \approx D_{1} x^{-\left[\frac{\beta+\gamma+2}{2}+\frac{\delta}{\alpha}\right]}+D_{2} e^{-\alpha x} x^{-\left[\frac{\beta+\gamma+2}{2}-\frac{\delta}{\alpha}\right]} \\
& =e^{-\frac{\alpha x}{2}} x^{-\frac{\beta+\gamma+2}{2}}\left[D_{1} e^{\frac{\alpha x}{2}} x^{-\frac{\delta}{\alpha}}+D_{2} e^{-\frac{\alpha x}{2}} x^{\frac{\delta}{\alpha}}\right] \\
& =C e^{-\frac{\alpha x}{2}} x^{-\frac{\beta+\gamma+2}{2}} \sin \left[-i \frac{\alpha x}{2}+i \frac{\delta}{\alpha} \ln x+\varphi\right]
\end{aligned}
$$

where $\varphi$ is the phase shift.

Using the parameters (12) in the above expression, the zero-order solution corresponding to the outgoing mode is given by

$$
\Phi_{l m} \approx \frac{A}{r} e^{i p r} x^{\frac{i M}{p}\left(\omega^{2}+p^{2}\right)} Y_{l}^{m}(\theta, \varphi) e^{-i \omega t},
$$

where $p=\sqrt{\omega^{2}-\mu^{2}}$.

In a perturbative approach, the expression (14) can be used as a zero order solution to compute the transitions amplitudes initiated by the linear and quadratic metric's contributions (Dariescu et al., 2017).

Thus, for the metric function (1) written as

$$
g_{00}=g_{00}^{S}+h(r),
$$

where

$$
g_{00}^{S}=1-\frac{2 M}{r}, h(r)=\gamma r-\lambda r^{2}<<1,
$$

the Eq. (8) can be put in the form

$$
D \Phi=V \Phi,
$$

with

$$
D=\frac{1}{r^{2}}\left\{\frac{\partial}{\partial r}\left[r^{2} g_{00}^{S} \frac{\partial}{\partial r}\right]+\Delta_{\theta, \varphi}\right\}-\frac{1}{g_{00}^{S}} \frac{\partial^{2}}{\partial t^{2}}-\mu^{2}
$$

and

$$
V=\frac{1}{r^{2}}\left\{\frac{\partial}{\partial r}\left[\left(\lambda r^{4}-\gamma r^{3}\right) \frac{\partial}{\partial r}\right]\right\}+\frac{\omega^{2}(\gamma-\lambda r) r^{3}}{(r-2 M)\left(r-2 M+\gamma r^{2}-\lambda r^{3}\right)} .
$$


Let us consider $\Phi=\phi+\chi$, where $\phi$ is the homogeneous solution (14), to the zero-order equation

$$
D \phi=0
$$

This can be used to compute the first-order transition amplitude,

$$
A=\int \bar{\phi}(x) V(x) \phi(x) d \Omega
$$

with the integration measure

$$
d \Omega=r^{2} d r \sin \theta d \theta d \varphi d t
$$

and the potential operator (17).

\section{Conclusions}

Even though the metric (1) is not a standard solution obtained in General Relativity, in the last years, it has received considerable attention. This is due to the fact that it offers an explanation for flattened galactic velocity curves and effectively deal with the interpretation of the observed declination from the Hubble law requiring dark energy.

In the metric (1), one may notice besides the Schwarzschild de-Sitter solution, the linear term $\gamma r$, which is a special feature of the fourth order theory and has consequences at galactic distance scales.

The integration constants $M, \gamma$ and $\lambda$ have been fixed by fitting the observed galactic rotation curves. The only free parameter in the MK metric is the mass to light ratios, $M / L$, of the luminous optical disk of each galaxy.

The simple metric expression (2), with $\gamma=\gamma_{0}$, can be used for a large range of galaxies, as for example the dwarf galaxies, where the parameter $N^{*} \gamma^{*}$ is very small compared to $\gamma_{0}$ and also, at their distance scale, the $\lambda$ term contribution is not significant. Contrary to the Schwarzschild solution, in the metric (2), the falling Newtonian term is competing with the rising linear one and a region of flatness can appear, at $r_{*}=2 M / \gamma$.

A main part of the paper is dealing with the Gordon equation describing a particle moving in the spacetime described by the metric function (1). Since the analytical solution can be derived only for the Schwarzschild metric, we have used a numerical approach. By inspecting the Fig. 1, one may notice that, contrary to the Schwarzschild case given in the Fig. 2, the absolute value of the wave function preponderantly remains constant as the radius increases. This agrees with the observed data of velocities for spiral galaxies. In both cases, the 
density of probability vanishes on the black hole horizon and has a maximum just outside the horizon.

For rangers of $r$ where the linear and quadratic terms can be treated as perturbations, one may use the homogeneous solution (14) to compute the first order transition amplitudes. Such investigations on the motions of particles in the spacetime endowed with the MK metric is providing a way to detect the presence of the global de Sitter-like component.

\section{REFERENCES}

Ahnen M.L. et al., MAGIC Collaboration, Indirect Dark Matter Searches in the Dwarf Satellite Galaxy Ursa Major II with the MAGIC Telescopes, JCAP, 03, 009 (2018).

Dariescu C., Dariescu M., Stelea C., The SO(3,1) $\times$ U(1)-Gauge Invariant Approach to Charged Bosons in Relativistic Magnetars, General Relativity and Gravitation, 49, 153 (2017).

Dariescu M.A., Dariescu C., Solutions for Massless Bosons in the Mannheim -Kazanas Space-Time, Astrophysics, 64, 283 (2021).

Mannheim P.D., O'Brien J.G., Fitting Galactic Rotation Curves with Conformal Gravity and a Global Quadratic Potential, Physical Review D, 85, 124020 (2012).

Mannheim P.D., Kazanas D., Exact Vacuum Solution to Conformal Weyl Gravity and Galactic Rotation Curves, The Astrophysical Journal, 342, 635-638 (1989).

Milgrom M., A Modification of the Newtonian Dynamics as a Possible Alternative to the Hidden Mass Hypothesis, The Astrophysical Journal, 270, 365-370 (1983).

O'Brien J. G., Mannheim P.D., Fitting Dwarf Galaxy Rotation Curves with Conformal Gravity, Monthly Notices of the Royal Astronomical Society, 421, 1273-1282 (2012).

Persic M., Salucci P., Rotation Curves of 967 Spiral Galaxies, The Astrophysical Journal Supplement Series, 33, 205-211 (1995).

Ronveaux A., Heun's Differential Equations, The Clarendon Press, Oxford University Press, New York (1995).

Rubin V.C., Thonnard N., Ford W.K., Rotational Properties of 21 SC Galaxies with a Large Range of Luminosities and Radii, FROM NGC $4605 / r=4 \mathrm{kpc} /$ to Ugc $2885 / r=122 \mathrm{kpc} /$, The Astrophysical Journal, 238, 471-487 (1980).

Slavyanov S.Y., Lay W., Special Functions, A Unified Theory Based on Singularities, Oxford Mathematical Monographs (2000).

Sofue Y., Dark Halos of M31 and the Milky Way, Publications of the Astronomical Society of Japan, 67, 75 (2015).

Vieira H., Bezerra V., Confluent Heun Functions and the Physics of Black Holes: Resonant Frequencies, Hawking Radiation and Scattering of Scalar Waves, Annals of Physics, 373, 28-42 (2016). 


\section{PERTURBAREA MIȘCĂRII KEPLERIENE ÎN \\ GALAXIILE SPIRALE \\ DESCRISE DE METRICA MANNHEIM-KAZANAS}

\section{(Rezumat)}

Scopul acestei lucrări este analiza ecuației Klein-Gordon pentru metrica Mannheim-Kazanas, care conține, pe lângă soluția Schwarzschild, un termen linear și unul pătratic. Această metrică oferă o explicație pentru curbele de rotație plate ale galaxiilor spirale, fără ipoteza materiei întunecate. Asupra particulei test, care orbitează centrul galaxiei, acționează nu numai masa galactică ci și componenta cosmologică de fond, care poate fi tratată ca o perturbație. 